\title{
Valoración de la aptitud laboral en trabajadoras sanitarias con cáncer de mama
}

\section{Labor capability assessment for health workers with breast cancer}

\section{Consuelo Rodrigo García-Pando, Cristina Goenaga Olaizola, Juan José Granados Arroyo}

Servicio de Prevención de Riesgos Laborales. Área 9. Servicio Madrileño de Salud. Madrid. España.

Recibido: 04-05-11

Aceptado: 28-07-11

\section{Correspondencia}

Consuelo Rodrigo García-Pando

Servicio de Prevención de Riesgos Laborales

Hospital Universitario Severo Ochoa

Avda. de Orellana, s/n

28911 LEGANÉS (Madrid). España.

Tfno: 914818000 ext. 8348

e-mail: crodrigo.hsvo@salud.madrid.org

\section{Resumen}

El cáncer de mama es el tumor más frecuente en las mujeres occidentales y afectará a una de cada ocho mujeres a lo largo de su vida. Su incidencia se ha incrementado pero la tasa de mortalidad ha descendido. El médico del trabajo debe valorar y adoptar las medidas de prevención adecuadas para atenuar los riesgos a los que puedan estar expuestos los trabajadores especialmente sensibles en el ámbito sanitario.

Nuestro objetivo es proponer un método para esta valoración así como las adaptaciones necesarias para el desempeño de su tarea.

Partimos de una revisión bibliográfica del cáncer de mama y se analizan los riesgos laborales del ámbito sanitario que pudieran suponer un agravamiento de las condiciones de salud de la trabajadora afectada. Exponemos un modelo de reconocimiento médico y valoración de la capacidad funcional adaptado a las secuelas más probables presentadas por las mujeres que han sido diagnosticadas de cáncer de mama, para proponer unos criterios de aptitud y recomendaciones de adecuación del puesto de trabajo.

Med Segur Trab (Internet) 2011; 57 (224) 210-223

Palabras clave: aptitud, cáncer de mama, trabajador especialmente sensible, ámbito sanitario

\begin{abstract}
Breast cancer is the most frequent tumour in occidental women and it will affect one in eight women throughout their lives. Over the years, incidence rate has increased, whereas, mortality rate has decreased. The labour physician has to assess and decide on the suitable prevention measures to reduce those labour risks to which especially sensitive health workers may be exposed in their labour environment.

Our objective is to propose a method for carrying out the assessment and the necessary adjustments for them to do their job.

We begin with a bibliographic review about breast cancer to analyze the risks found in the health work environment that could worsen health conditions of the affected worker. We expose a model of medical examinations and assessment of the functional capacity, adapted to the most usual sequels shown up in
\end{abstract}


women that have been diagnosed with breast cancer. We can then propose some aptitude criteria and work advice to improve health working conditions.

Med Segur Trab (Internet) 2011; 57 (224) 210-223

Key words: aptitude, breast cancer, especially sensitive worker, health work environment. 


\section{INTRODUCCIÓN}

El artículo 25 de la Ley 31/1995 de Prevención de Riesgos Laborales (LPRL) establece que el empresario garantizará de manera específica la protección de los trabajadores que, por sus propias características personales o estado biológico conocido, incluidos aquellos que tengan reconocida la situación de discapacidad física, psíquica o sensorial, sean especialmente sensibles a los riesgos derivados del trabajo.

El Real Decreto 39/1997, Reglamento de los Servicios de Prevención (RSP) establece como función del médico del trabajo estudiar, valorar y proponer las medidas adecuadas para prevenir los riesgos a los que puedan estar expuestos los trabajadores especialmente sensibles, en nuestro caso por cáncer de mama.

Una vez conocidos los riesgos existentes y las demandas del puesto de trabajo, el médico del trabajo debe valorar la aptitud del trabajador para este puesto, teniendo en cuenta las características o circunstancias personales o de enfermedad física o psíquica que le hagan más vulnerable. Como consecuencia de ello debe recomendar al empresario la adecuación de tareas o el cambio de puesto de trabajo.

El cáncer de mama es el tumor más frecuente en las mujeres occidentales ${ }^{1}$ y afectará a una de cada ocho mujeres a lo largo de su vida ${ }^{2}$. Solo el $1 \%$ de todos los cánceres de mama ocurren en varones ${ }^{3,4}$.

La incidencia del cáncer de mama en los países desarrollados se ha incrementado, sin embargo la tasa de mortalidad ha descendido, se cree que debido al diagnóstico precoz y a un tratamiento más eficaz ${ }^{5,6}$.

\section{MATERIAL Y MÉTODOS}

Se realiza una revisión bibliográfica sobre la situación actual del cáncer de mama en relación con el trabajo y discapacidades en las bases de datos: OSH UPDATE, IBECS, CISDOC y PUBMED.

En OSH UPDATE (19 bases de datos en salud laboral), IBECS (Índice Bibliográfico Español en Ciencias de la Salud), CISDOC (base de datos de la Organización Internacional del Trabajo sobre Seguridad y Salud Laboral) se combinan para la búsqueda las palabras claves breast, cancer, prevalencia, mama, aptitud, trabajadores especialmente sensibles, disability, job análisis, enabling options, disabled workers, vulnerable, disadvantaged.

En pubmed la estrategia de búsqueda es: 1 fitness for work; 2 fitness for duty; 3 fitness to work; 4 occupational fitness; 5 fitness for employment; 6 fitness for task; 7 job fitness; 8 preemployment medical examination; 9 preemployment examination; 10 periodical medical examination; 111 or 2 or 3 or 4 or 5 or 6 or 7 or 8 or 9 or 10 ; 12 assessment*; 13 evaluation*; 14 decision*; 1512 or 13 or 14; 11 end 15;

En Uptodate se actualiza la información referente a cáncer de mama.

Se consulta con especialistas en Ginecología y Rehabilitación del Hospital Universitario Severo Ochoa de Leganés, Madrid (España) y con profesorado del XXII Diploma en Valoración Médica de Incapacidades (Curso 2009-2010) de la Escuela Nacional de Medicina del Trabajo ubicada en Madrid (España), como asesoramiento en los temas de su competencia.

Otra documentación consultada:

- Valoración de la incapacidad temporal y permanente en las enfermedades oncológicas realizada por la Inspección Médica Española.

- Estrategia en Cáncer del Sistema Nacional de Salud. Plan de Calidad para el Sistema Nacional de Salud en España. 
- Legislación española aplicable como la Ley de Prevención de Riesgos Laborales 31/1995. Reglamento de los Servicios de Prevención RD 39/1997. Directiva 89/391/CEE. Estatuto de los trabajadores. Constitución Española. Convenio $\mathbf{n}^{\circ}$ 159 de la Organización Internacional del Trabajo. Recomendaciones $\mathrm{n}^{\circ} 99$ y $\mathrm{n}^{\circ}$ 168 de la Organización Internacional del Trabajo. OIT:Recomendaciones 99 y 168 y convenio $n^{\circ} 159$.

- Guias de practica clínica en relación con cáncer de mama: Fisterra, Oncoguía en cancer de mama. 2005. Comundad Valenciana (España), OMS (The breast health câncer initiative (Wiley Interscience:Guidelines for international breast health and cancer control-implementation), American Society of Clinical Oncology (ASCO), European Society of Medical Oncology (ESMO), Steering Committee on Clinical Practice Guide line for the care and Treatment of Breast Cancer of Health Canada, Instituto Nacional del Cancer. USA.

También se analizan los riesgos laborales presentes en el ámbito sanitario, destacando aquellos que pudieran suponer un agravamiento de las condiciones de salud de la trabajadora con cáncer de mama. Posteriormente exponemos un modelo de reconocimiento médico y valoración de la capacidad funcional adaptado a las secuelas más probables presentadas por las mujeres que han sido diagnosticadas de cáncer de mama. Por último se exponen unos criterios de aptitud y recomendaciones de adecuación del puesto de trabajo.

\section{RESULTADOS}

\section{El cáncer de mama}

\section{Mortalidad}

En España, en 2007, el cáncer de mama fue la primera causa de muerte por cáncer en mujeres (5.948 fallecimientos por cáncer de mama de 38.995 por tumores en mujeres, 15,2\%). En 2005, la tasa de mortalidad cruda por cáncer de mama fue de 26.0 por 100.000 y la tasa de mortalidad estandarizada por edad por cáncer de mama de 12,9 por $100.000^{7}$.

La mortalidad por cáncer de mama en España comienza a descender en el año 1992, a un ritmo del $2 \%$ anual presentando una mayor supervivencia los grupos de edad más jóvenes.

\section{Incidencia}

En España, la tasa de incidencia estimada ajustada por edad para el año 2006 era de 93,6 casos por 100.000, una de las más bajas de la $\mathrm{UE}^{1,3}$.

La mayor incidencia se observa en las mujeres de clase social más elevada. Determinadas profesiones - profesoras, farmacéuticas, trabajadoras sanitarias, empleadas de la industria química, trabajadoras de telefonía y radio y peluqueras- muestran también una incidencia mayor, aunque es difícil deslindar la influencia de factores específicamente ocupacionales.

\section{Prevalencia}

Se estima que actualmente en España existirían 70.000 mujeres diagnosticadas de cáncer de mama en los últimos 5 años ${ }^{1}$.

\section{Supervivencia}

Se sitúa en un 83\% a los cinco años del diagnóstico, significativamente más alta que la media europea, cercana al $79 \%{ }^{8}$. 
En el estudio EUROCARE-3 realizado entre 1990-1994 con registros procedentes de País Vasco, Gerona, Granada, Mallorca, Navarra y Tarragona la tasa de supervivencia a los 5 años era del $78 \%{ }^{9}$.

Evolución supervivencia en España de pacientes con cáncer de mama

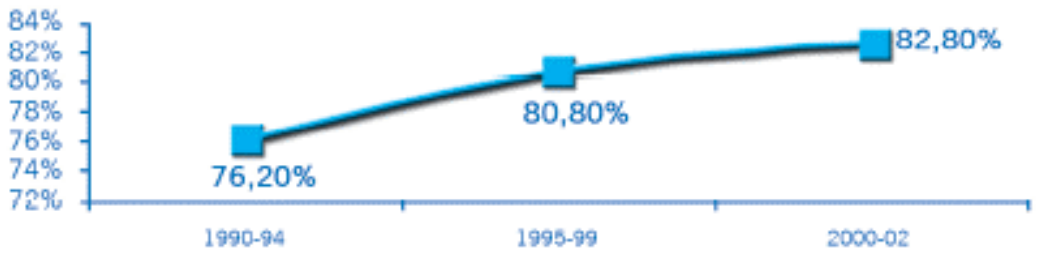

Fuente: Estudio Eurocare 4 (The Lancet Oncology 2007 vol. 8 No 9:773-783

y The Lancet Oncol 2007 vol.8 No 9: 784-796). Gráfica elaborada por SEOM

La supervivencia a los 5 años varía según el estado del cáncer de mama en el momento del diagnóstico, siendo del 98\% cuando el tumor está localizado, 84\% si está delimitado regionalmente y $27 \%$ si hay afectación a distancia.

El cáncer de mama no produce síntomas en las fases precoces de la enfermedad, la presencia de síntomas atribuibles al cáncer de mama suele indicar una enfermedad avanzada de nivel o con metástasis.

Los signos y síntomas pueden ser distintos en cada persona. Una mujer puede descubrir que tiene cáncer de mama por medio de una mamografía de rutina. El diagnóstico se establece por el estudio anatomo-patológico. Las pruebas complementarias son necesarias para establecer el estadio y el diagnóstico de complicaciones.

Para la valoración clínica y pronóstica del cáncer de mama se deben tener en cuenta las clasificaciones que tanto el American Joint Comite On Cancer ${ }^{10}$ (AJCC) como la internacional Union Against Cáncer ${ }^{11}$ (UICC) proponen como estadios de la enfermedad. En ellos se tienen en consideración tanto la extensión del tumor como la histología, inmunohistoquímica, afectación ganglionar y metástasis a distancia.

\begin{tabular}{|c|c|c|c|}
\hline AJCC & & IUAC & \\
\hline Estadío 0 & Tis & N0 & M0 \\
\hline Estadío I & $\mathrm{T} 1$ (incluye $\mathrm{T} 1 \mathrm{mic}$ ) & N0 & M0 \\
\hline \multirow[t]{3}{*}{ Estadío IIA } & T0 & N1 & M0 \\
\hline & T1 (incluye T1 mic) & N1 & M0 \\
\hline & T2 & N0 & M0 \\
\hline \multirow[t]{2}{*}{ Estadío IIB } & $\mathrm{T} 2$ & N1 & M0 \\
\hline & T3 & No & M0 \\
\hline \multirow[t]{4}{*}{ Estadío IIIA } & T0 & $\mathrm{N} 2$ & M0 \\
\hline & T1 (incluye T1 mic) & $\mathrm{N} 2$ & M0 \\
\hline & T2 & $\mathrm{N} 2$ & M0 \\
\hline & T3 & $\mathrm{N} 1, \mathrm{~N} 2$ & M0 \\
\hline \multirow[t]{2}{*}{ Estadío IIIB } & T4 & Cualquier N & M0 \\
\hline & Cualquier $\mathrm{T}$ & N3 & M0 \\
\hline Estadío IV & Cualquier T & Cualquier N & M1 \\
\hline
\end{tabular}

Posteriormente hemos de analizar ciertos aspectos fundamentales para establecer un pronóstico vital, funcional y laboral. 
Los factores pronósticos más importantes son el tamaño del tumor, la afectación ganglionar, la existencia de receptores hormonales, sobre expresión del gen HER2, las secuelas derivadas del tratamiento y la comorbilidad de la paciente.

Otros factores pronósticos serán dependientes del tumor como la localización de éste, tipo histológico, grado de diferenciación, extensión e infiltración a tejidos vecinos; o dependientes del paciente como la edad, estado funcional (el índice Karnofsky es el principal determinante de la evolución de la enfermedad), comorbilidad con enfermedades cardíacas, renales, pulmonares, hepáticas o neurológicas o existencia de tratamientos concomitantes $^{12,13}$.

\section{Tratamiento y sus efectos secundarios}

El tratamiento es complejo y variado. Paciente y médico tienen la responsabilidad de conocer y elegir la opción más adecuada de las alternativas posibles: tratamiento de la enfermedad local con cirugía, radioterapia o ambos y tratamiento de la enfermedad sistémica con quimioterapia citotóxica, tratamiento hormonal, tratamiento biológico o una combinación de todos ellos ${ }^{6}$.

La elección se hace en función de diferentes factores: aspectos clínicos y patológicos del tumor primario, afectación ganglionar axilar, contenido de receptores hormonales, situación HER2, presencia de metástasis, comorbilidad, edad y menopausia.

1. Tratamiento neoadyuvante (quimioterapia preoperatoria) se realiza en mujeres con tumores HER2 positivo siendo recomendable el uso del anticuerpo monoclonal trastuzumab por su especificidad para este receptor Los taxanos constituyen una opción a tener en cuenta en la enfermedad avanzada 5 .

\section{Cirugía}

Dependiendo de la técnica practicada, puede provocar afectación funcional o estética ${ }^{2,14}$.

El valor de la linfadenectomia axilar es fundamentalmente pronóstico y está siendo sustituida por la biopsia del ganglio centinela. El objetivo es detectar, extirpar y estudiar el ganglio que tiene más probabilidad de estar afectado, evitando en caso de ausencia de infiltración las secuelas asociadas a linfadenectomía.

Los problemas principales dentro del tratamiento de las mujeres intervenidas de cáncer de mama, son el linfedema, la capsulitis adhesiva, el dolor secundario a lesión de partes blandas y el dolor neuropático, que merman en gran medida la capacidad funcional y su reincorporación social, familiar y laboral ${ }^{15}$.

El 23\% del linfedema secundario se produce en los dos años siguientes a la cirugía y el $41 \%$ tras cirugía y radioterapia. El periodo de mayor riesgo de aparición se sitúa en los 6 meses posteriores al tratamiento.

El linfedema es el resultado de un fallo en el sistema linfático en el que el volumen de linfa excede la capacidad de drenaje de los linfáticos axilares, es un edema crónico producido por la acumulación de un fluido rico en proteínas en el miembro superior. La mujer con linfedema puede experimentar dolor, inflamación del brazo, tirantez, pesadez, infecciones cutáneas recurrentes y un deterioro en la calidad de vida. En muchos casos será leve pero en otros tendrá un linfedema clínicamente significativo que limitará su capacidad laboral.

Grados de linfedema:

- G0: miembro con ligero aumento de volumen que desaparece con reposo nocturno.

- G1: edema que regresa parcialmente. Sensación de pesadez.

- G2: linfedema franco que no regresa espontáneamente con posición de drenaje.

- G3: edema severo o irreversible (fibrolinfedema)

- G4: Elefantiasis. Paquidermitis y verrucosis linfoestética. 
El tratamiento del linfedema estaría justificado ante síntomas de pesadez, tirantez o hinchazón del brazo afectado, con una diferencia mayor de $2 \mathrm{cms}$ en la circunferencia medida en cuatro puntos. Las medidas conservadoras no farmacológicas constituyen en la actualidad el principal tratamiento ${ }^{16}$.

La terapia física tiene como objeto la movilización de la linfa acumulada en el tejido celular subcutáneo y modificar la estructuración de tabiques intermoleculares consecuencia de la evolución natural de las fibras de colágeno sometidas a linfedema ${ }^{17}$.

La capsulitis adhesiva y el dolor secundario a lesión de partes blandas se produce con una frecuencia del $20 \%$ en las pacientes en las que se realiza ganglio centinela y hasta en el $72 \%$ de vaciamientos axilares. La mayor disminución del rango de movimiento articular está relacionada con la asociación de mastectomía radical con vaciamiento ganglionar y radioterapia. Esta limitación aparece entre los tres meses y los dos primeros años tras tratamiento.

El dolor neuropático está presente entre el 23\% y 55\% de las pacientes y suele asociarse a síntomas neuropáticos como alodinia, hiperalgesia, hipoestesia, anestesia, etc. Tiene como origen lesiones neuromusculares (secundarias a cirugía de vaciamiento ganglionar, postirradiación, plexopatias infiltrativas, etc.)

\section{Radioterapia}

La radioterapia complementaria reduce el número de recidivas loco-regionales y mejora la supervivencia global. La radioterapia ha demostrado una reducción del 30\% del riesgo de recurrencia 5 .

La piel en la zona tratada con radiación puede enrojecerse, secarse, hacerse sensible y con comezón, y el seno puede sentirse pesado y estirado. Dichos problemas desaparecen generalmente con el tiempo ${ }^{18}$.

Los efectos tóxicos tardíos de la radioterapia, aunque poco comunes, pueden incluir neumonitis por radiación, complicaciones cardiacas, edema de brazo, plexopatía braquial y riesgo de neoplasias malignas secundarias. Con las técnicas modernas se ha minimizado la radiación profunda.

El tratamiento con radioterapia axilar en las pacientes sometidas a disección axilar puede aumentar el riesgo de edema de brazo de 2 a 10\%.La radioterapia ha mejorado en ajuste de dosis y localización por lo que se han minimizado los efectos secundarios.

En la actualidad también se ha minimizado el riesgo de radiación profunda del miocardio.

\section{Tratamiento adyuvante}

El tratamiento adyuvante en cáncer de mama precoz, tanto hormonal como con quimioterapia, reduce el riesgo de recidiva y de muerte (independientemente de edad, afectación ganglionar y sensibilidad hormonal) de las pacientes pre y posmenopáusicas.

La quimioterapia adyuvante se relaciona con varios efectos tóxicos bien caracterizados que varían según los fármacos utilizados. Entre los más comunes se incluyen nauseas y vómitos, mielodepresión, alopecia y mucositis.

Otros menos comunes pero graves son: la insuficiencia cardiaca (si se usa una antraciclina), episodios tromboembólicos y menopausia prematura.

Se debe tomar en cuenta, en la selección del régimen quimioterápico, el potencial de cardiotoxicidad inducida por la doxorubicina (antraciclina). Los factores reconocidos de riesgo de toxicidad cardiaca incluyen: edad avanzada, radioterapia previa dirigida a la pared torácica, exposición previa a antraciclina, hipertensión, diabetes o enfermedad cardiaca subyacente.

El uso de quimioterapia combinada con regímenes que contienen antraciclina y especialmente los que contienen una dosis más alta de ciclofosfamida, parece que tienen un mayor riesgo de desarrollar una leucemia aguda. 


\section{Terapia endocrina}

El Tamoxifeno presenta efectos beneficiosos añadidos por su acción agonista parcial estrogénica: reducción de la pérdida de masa ósea y la disminución de cLDL.

Entre sus inconvenientes están la aparición de síntomas vasomotores (sofocos), sequedad vaginal, aumento de peso, cefalea, accidentes tromboembólicos (trombosis venosa profunda y embolia pulmonar), cataratas, quistes benignos de ovario y sobre todo aumento de riesgo de cáncer de endometrio.

En mujeres premenopáusicas puede ser necesaria la utilización de análogos de la hormona liberadora de gonadotropinas (GnRHa) como la goserelina o triptorelina que, producen una deprivación de la función ovárica generalmente reversible.

En mujeres posmenopáusicas con positividad en los receptores hormonales se debe usar un inhibidor de la aromatasa (anastrazol, exemestane o letrozole) como terapia adyuvante ya que ha demostrado buenos resultados en las tasas de supervivencia. Los efectos secundarios más frecuentes del anastrazol ${ }^{19}(>10 \%)$ son los trastornos vasomotores (sofocos, principalmente de naturaleza leve o moderada), si bien, son también frecuentes $(>1 \%$ y $<10 \%$ ) la astenia (principalmente de naturaleza leve o moderada) o las alteraciones músculo-esqueléticas, tejido conjuntivo y óseo como dolor/rigidez en las articulaciones también de naturaleza leve o moderada.

Los efectos secundarios del exemestane incluyen artralgias, diarrea, hipertensión, fracturas, artritis, dolores músculo-esqueléticos, síndrome del túnel carpiano, insomnio u osteoporosis.

\section{Terapia inmunológica}

En mujeres con tumores HER2 positivo es recomendable el uso del anticuerpo monoclonal trastuzumab por su especificidad para este receptor. Unido a la quimioterapia mejora la tasas de respuesta y de supervivencia.

El uso de trastazumab está asociado a cardiotoxicidad ${ }^{5}$. La forma de presentación más habitual es un descenso asintomático de la fracción de eyección ventricular izquierda (FEVI). Una disminución de más de 10 puntos o hasta el 55\% de FEVI, que revierte con la interrupción del tratamiento ${ }^{14}$. La cardiotoxicidad se ve potenciada con el uso previo o simultaneo de antraciclinas.

Lapatinib se presenta como tratamiento inmunológico complementario al trastuzumab.

\section{Otros tratamientos}

\subsection{Osteoporosis, osteopenia y metástasis óseas}

Las mujeres premenopáusicas con insuficiencia ovárica secundaria y quimioterapia adyuvante y las posmenopáusicas en tratamiento con inhibidores de la aromatasa tienen aumentado el riesgo de osteoporosis, es conveniente vigilar la salud ósea en este grupo de mujeres así como el tratamiento sustitutivo con calcio y vitamina $\mathrm{D}$, uso de bifosfonatos y examen dental previo al uso de éstos ${ }^{5,6}$.

Las metástasis óseas aparecen en el $65-75 \%$ de las pacientes con cáncer avanzado ${ }^{7}$. Los bifosfonatos se deben utilizar de manera rutinaria en combinación con el tratamiento sistémico en las pacientes con metástasis óseas.

Se ha visto un riesgo aumentado de osteomielitis mandibular (5,48 eventos por 100 pacientes tratados) con el uso de bifosfonatos intravenosos. También se ha documentado la aparición de hipofosfatemia e hipocalcemia.

\subsection{Tratamiento psicológico}

La evaluación de la presencia de síntomas psicológicos de adaptarán a las circunstancias especiales de cada paciente. No se recomienda la utilización rutinaria de cuestionarios para la detección de este tipo de problemas salvo que existan factores de riesgo específicos. Es adecuado facilitar la inclusión en un grupo de intervención psicológica en los casos en los que se necesite 5 . 
Algunos inhibidores de la recaptación de serotonina disminuyen la formación de endoxifeno, un metabolito activo del tamoxifeno. Sin embargo, citalopram y velaflaxina parecen tener un impacto mínimo en el metabolismo del tamoxifeno. La importancia clínica de estos hechos es desconocida ${ }^{6}$.

\subsection{Tratamiento rebabilitador}

Los ejercicios de brazo y hombro se deben iniciar lo antes posible tras la operación y las pacientes deben continuar con ejercicios de manera continuada.

Resumen de las secuelas más frecuentes en mujeres diagnosticadas y tratadas de cáncer de mama:

- Algias locoregionales de partes blandas.

- Linfedema tras linfadenectomía.

- Trastornos del ánimo. S depresivo.

- Capsulitis adhesiva hombro.

- Dolor neuropático.

- Radiculopatía.

- Patología cicatricial, dermatitis.

- Cardiotoxicidad secundaria a antraciclina y/o trastuzumab.

- Síntomas vasomotores, cataratas, osteoporosis.

- Accidentes tromboembólicos.

— Raras: neumonitis, mielodepresión.

\section{Estructura y contenido de la valoración}

La trabajadora deberá ser evaluada cuando se reincorpore a trabajar tras ausencia prolongada por motivos de salud (Reglamento de los Servicios de Prevención. Artículo 37.3.b.2).

Proponemos como método de valoración aquel que combine el ajuste trabajopersona y la prevención de riesgos laborales. Así debemos analizar tanto las demandas del trabajo, la capacidad de la trabajadora para satisfacer éstas, y los riesgos que pueden derivarse de la realización del trabajo.

Requisitos para esta valoración:

1. Descripción de las tareas esenciales y no esenciales de su puesto de trabajo y evaluación de riesgos laborales.

2. Valoración de la capacidad funcional de la trabajadora diagnosticada de cáncer de mama, es decir, de lo que puede hacer y lo que no puede hacer. Reconocimiento médico laboral.

3. Definir su grado de aptitud y especificar las necesidades concretas de adecuación del puesto de trabajo o la necesidad de cambio de puesto de trabajo.

\section{Riesgos laborales}

A partir de la evaluación de riesgos laborales realizada por el Equipo Técnico del Servicio de Prevención de Riesgos Laborales observamos los riesgos en el ámbito sanitario que pueden interferir con la especial sensibilidad de las trabajadoras con cáncer de mama. Así tenemos:

- Riesgos químicos:

- Citostáticos.

- Óxido de etileno.

- Formaldehído.

- Agentes anestésicos inhalados. 
- Compuestos químicos irritantes de vías respiratorias (R37).

- Compuestos químicos cancerígenos o genotóxicos (R45/46/49).

- Riegos físicos:

- Radiaciones ionizantes

- Radiaciones no ionizantes.

- Riesgos biológicos.

- Riesgos ergonómicos y psicosociales:

- Posturas forzadas.

- Manipulación manual de cargas.

- Movimientos repetidos.

- Turnicidad, nocturnidad.

- Demandas psíquicas de las tareas.

- Manejo de maquinaria peligrosa.

- Conducción de vehículos.

Reconocimiento médico laboral (valoración de la capacidad funcional en relación con el estado clínicol

1. Fecha de valoración.

2. Datos de filiación: nombre, fecha de nacimiento, sexo, domicilio, categoría, puesto de trabajo, centro.

3. Historia laboral: periodos trabajados, empresa, categoría, puesto de trabajo, riesgos laborales existentes.

4. Antecedentes laborales: accidentes de trabajo, enfermedades profesionales, enfermedades relacionadas con el trabajo, periodos de incapacidad laboral, cambios de puesto de trabajo en relación con motivos de salud.

5. Antecedentes familiares.

6. Antecedentes personales: patologías previas, alergias, hábitos.

7. Enfermedad actual:

7.1. Situación alegada por el trabajador sobre su estado actual y posibilidades de desarrollar sus tareas habituales.

7.2. Anamnesis sobre la enfermedad que motiva esta valoración:

- Estado de la enfermedad.

- Tratamientos a los que ha sido sometido, si sigue en tratamiento en la actualidad o si está pendiente de tratamientos posteriores.

- Aporte de informes de especialistas y pruebas complementarias (ecocardiograma, prueba de esfuerzo, electromiograma, análisis clínicos, espirometría, test de fatiga respiratoria...)

7.3. Exploración:

a. General: Escalas de Karnofsky, AMC, Zubrod y ECOG.

b. De su estado psico-afectivo. Entrevista clínica, ya que solo se aconseja la realización de test diagnósticos si se observan alteraciones, principalmente trastornos del ánimo (test de Goldberg, GHQ-28).

c. Exploración física general con especial detenimiento en:

Aparato musculoesquelético:

- conocer si afecta al miembro dominante.

- realizar inspección general (atrofia muscular, asimetrías, existencia de linfedema).

- palpar de puntos dolorosos.

Valoración de la aptitud laboral en trabajadoras sanitarias con cáncer de mama Consuelo Rodrigo García-Pando, Cristina Goenaga Olaizola, Juan José Granados Arroyo 
- valorar la existencia de inflamación.

- arcos de movilidad activa, pasiva y contrarresistencia (escala de Daniels).

- exploración neurológica (motora, sensitiva, reflejos osteotendinosos).

- maniobras especiales de exploración.

Sistema cardiovascular:

- Exploración clínica: coloración, edemas, circulación periférica, presión arterial, frecuencia cardíaca, auscultación cardíaca, palpación de pulsos periféricos.

- Ecocardiograma: estudio morfológico y funcional, valora la fracción de eyección ventricular izquierda (FEVI).

- Normal: FEVI > 50\%.

- Leve: FEVI 49-40\%.

- Moderada: FEVI 39-30\%.

- Grave: FEVI <30\%.

- Ergometría:

- Prueba de esfuerzo negativa: >12 METS. Normal.

- Prueba de esfuerzo negativa: 10-12 METS.

- Prueba de esfuerzo positiva a partir del $5^{\circ}$ minuto o 7-9 METS. Prueba de esfuerzo positiva precoz y/o 4-6 METS.

Sistema respiratorio:

- Auscultación.

- Disnea: escala de Borg modificada (0 puntos sin disnea, 3 puntos disnea moderada, 5 puntos disnea severa, 10 puntos máxima disnea).

- Pulsioximetría: normal $>95 \%$. Si $<90 \%$ indica $\mathrm{PO} 2<60 \mathrm{mmHg}$.

- Gasometría: $\mathrm{PaO} 2<60 \mathrm{mmHg}$ insuficiencia respiratoria.

- Espirometría: Intensidad FVC, FEV1 o ambos expresado como \% del valor de referencia.

- Ligera hasta el $65 \%$.

- Moderada entre el $64 \%$ y el $50 \%$.

- Intensa entre el $49 \%$ y el 35\%.

- Muy intensa $<35 \%$.

- Test de difusión o transferencia de monóxido de carbono (TLCO o DLCO). Estudia el intercambio de gases a través de la membrana alveolo capilar:

- Normal: $100-80 \%$ DLCO.

- Ligero: 79-60\% DLCO.

- Moderado: 59-40\% DLCO.

- Grave: $<40 \%$ DLCO.

- Ergometría: Cuando la situación clínica no es concordante con la exploración funcional. Informa del consumo máximo de oxígeno del individuo (VO2 máx) y METS (consumo metabólico basal de oxígeno):

- Normal: 25VO2 (>7.1 METS).

- Disminución leve 25-50 (>7.1-5.7 METS).

- Disminución moderada 20-15 (5.7-4.3 METS).

- Disfunción severa <15 (<4.3 METS).

Valoración de la aptitud laboral en trabajadoras sanitarias con cáncer de mama Consuelo Rodrigo García-Pando, Cristina Goenaga Olaizola, Juan José Granados Arroyo 
Piel y mucosas:

- Inspección: coloración, integridad, estado de las cicatrices, existencia de hematomas, celulitis o edemas.

- Linfedema: analizar la consistencia, reversibilidad con posición de drenaje y volumen. Para ello se realiza una medición en cuatro puntos y se compara con el otro brazo. Los puntos a medir son los perímetros de la mano a nivel de $1 / 3$ distal de metacarpo, muñeca, antebrazo y brazo (los dos últimos a $12 \mathrm{~cm}$ del epicóndilo).

8. Diagnóstico clínico.

9. Valoración y pronóstico de las secuelas.

\section{Valoración de la aptitud para desempeñar su puesto de trabajo}

Con el análisis realizado se ha de emitir un juicio clínico laboral que la empresa debe conocer y que irá referido a alguna de las siguientes categorías:

APTA: Cuando una vez realizado el examen de salud específico, presenta una capacidad funcional sin limitaciones para las tareas esenciales de su puesto de trabajo. Supone una valoración funcional con Karnofsky por encima del $90 \%$ y sin deterioro funcional residual.

NO APTA: cuando su situación clínica no responda a las exigencias psicofísicas del puesto de trabajo, suponiendo un riesgo para si misma, los demás trabajadores o terceras personas. Estaría contraindicada la exposición a radiaciones ionizantes, citostáticos, formaldehído, oxido de etileno u otros productos químicos con potencial cancerígeno o genotóxicos (R45/46/49) por su posible efecto sobre el material genético de la trabajadora.

APTA CONDICIONAL: se puede incorporar pero solo si se cumplen determinadas condiciones en relación con la adaptación de tareas, equipos o lugar de trabajo. Estas condiciones han de ser especificadas y pueden ser temporales o permanentes.

Para valorar la aptitud de forma genérica partimos de las alteraciones funcionales por aparatos mas frecuentemente encontradas en trabajadoras diagnosticadas de cáncer de mama y proponemos unas adecuaciones de tareas en base a ello.

\section{Sistema músculo esquelético}

Conocer si existe limitación en arcos de movilidad, fuerza muscular, sensibilidad o destreza manual valorar las tareas que impliquen manejo manual de cargas, posturas mantenidas o forzadas, movimientos repetidos, digito prensión, trabajos de precisión. Es importante saber si la afectación corresponde al miembro dominante o no.

\section{Sistema circulatorio y piel}

En caso de presentar cicatrices retráctiles, valorar la movilidad según criterios del apartado anterior.

Si se objetiva linfedema, valorar su grado de afectación. Debe evitar realizar movimientos repetitivos, manejo manual de cargas, inmersión prolongada de manos en agua, exposición en proximidad a fuentes de calor (entre ellas radiaciones no ionizantes) así como protegerse de microtraumatismos especialmente aquellos con riesgo biológico. Un linfedema franco (grado 2 o más) imposibilitaría la realización de movimientos manuales de precisión.

Si existen antecedentes de tromboembolismo debe evitar posturas estáticas y bipedestación prolongada.

\section{Estado psicoafectivo}

Si presenta trastorno del ánimo debemos conocer la medicación pautada y valorar las exigencias de la tarea, toma de decisiones, contacto con pacientes (la enfermedad o la muerte), ritmo de trabajo (apremio y autonomía en la organización de tareas), turnicidad y nocturnidad. 
Si recibe tratamiento con ansiolíticos y/o antidepresivos valorar tareas que impliquen especial peligrosidad por el manejo de aparatos, trabajos de precisión, sistemas de alerta, conducción, etc.

\section{Estado inmunitario}

El riesgo de enfermedad profesional por exposición a agentes biológicos será valorado en relación con situaciones de inmunosupresión.

\section{Función cardíaca}

Si se constata una FEVI >50\% y/o 10-12 METS, no realizar cargas físicas extenuantes o competitivas.

Una prueba de esfuerzo positiva a partir del $5^{\circ}$ minuto o 7-9 METS implicaría no realizar requerimientos físicos de mediana y gran intensidad.

En caso de una prueba de esfuerzo positiva precoz y/o 4-6 METS, solo sería apta para actividades muy sedentarias.

\section{Función respiratoria}

Si presenta alteración leve no debe realizar carga física extenuante. Si la alteración es moderada estará limitada para requerimientos físicos de mediana-gran intensidad y permanencia en ambientes de constatada contaminación aérea.

Ante una alteración de la función respiratoria evitar la exposición a compuestos químicos irritantes de las vías respiratorias (R37).

\section{CONCLUSIÓN}

La mujer que ha sido diagnosticada de cáncer de mama, una vez superado el tratamiento, puede ser perfectamente apta para su reincorporación a la vida laboral.

Los tratamientos actuales han avanzado de tal modo que empieza a contemplarse al paciente oncológico como un enfermo crónico, en el que el tratamiento contiene y atenúa la progresión sintomática, si bien los efectos secundarios del mismo, pueden comprometer la capacidad laboral.

Es necesario el diseño de programas de valoración de la aptitud para trabajar en los cuales se ponga de manifiesto la base científica para la toma de decisiones.

\section{AGRADECIMIENTOS}

A la doctora María José Ruiz Figueroa, Jefa del Servicio de Prevención de los Servicios Centrales Ministerio de Sanidad y Política Social de Madrid por su inestimable ayuda en la elaboración de este trabajo.

A la Escuela Nacional de Medicina del Trabajo de Madrid por su dedicación a la mejora de nuestra especialidad.

\section{REFERENCIAS BIBLIOGRÁFICAS}

1. Cabanes Domenech A, Pérez-Gómez B, Aragonés N, Pollán M, López-Abente G. La situación del cáncer en España. 1975-2006. [monografía en internet]. Madrid: Área de Epidemiología Ambiental y Cáncer. Centro Nacional de Epidemiología. Instituto de Salud Carlos III. Ministerio de Ciencia e Innovación; junio 2009 [acceso 14/5/2010]. Disponible en: http://www.isciii.es/htdocs/pdf/epicancerjunio2009.pdf

2. Medline Plus [sede web]. Bethesda:Biblioteca Nacional de Medicina de EE. UU. y los Institutos Nacionales de la Salud.23/3/2010 [acceso 27/3/2010]. Cáncer de mama. Disponible en: http://www.nlm. nih.gov/medlineplus/spanish/ency/article/000913.htm 
3. Sasco AJ; Lowenfels AB; Pasker-de Jong P. Review article: epidemiology of male breast cancer. A metaanalysis of published case-control studies and discussion of selected aetiological factors. Int J Cancer 1993 Feb 20; 53(4):538-49.

4. Plan Oncológico de la Comunidad Valenciana. : Conselleria de Sanitat, Comunitat Valenciana; 2007 [Consultado el 27/3/2010]. Disponible en: http://ppc.cesga.es/files/documentacion/guias/valencia_ oncoguia.pdf

5. Viana Zulaica, C. Cáncer de mama. Guías clínicas. [monografía en internet]. La Coruña: Fisterra.com; 2007 [Actualizado 26/09/2007] Consultado el 27/3/2010. Disponible en: http://www.fisterra.com/ guias2/mama.asp

6. Natinonal Comprehensive Cancer Network. NCCN Clinical Practice Guidelines in Oncology. Breast Cancer. NCCN V1; 2009.

7. Sociedad Española de Oncología Médica. El cáncer en España.com. España: Sociedad Española de Oncología Médica. [consultado el 27/3/2010]. Disponible en: http://www.seom.tv/index/SEOMTV/ diamundialcancer2010_files/cancer.pdf

8. Pollan M, García-Mendizábal M J, Pérez Gómez B, Aragonés N, Lope V, Pastor R, Ramis R, Fernandez P. Situación epidemiológica del cáncer de mama en España. Psicooncología. Vol. 4, Núm. 2-3, 2007, pp. 231-248.

9. Plan de Calidad para el Sistema Nacional de Salud. Estrategia en Cáncer del Sistema Nacional de Salud [monografía en Internet]. Madrid: Ministerio de Sanidad y Consumo. Centro de Publicaciones; 2006 [acceso 21/3/2010]. Disponible en: http:/www.msc.es/organizacion/sns/planCalidadSNS/pdf/ excelencia/cancer-cardiopatia/CANCER/opsc_est1.pdf.pdf

10. AJCC Cancer Stagin Manual. Chicago, Illinois: Springer-Verlag New York. Sixth edition (2002) (www. Cancerstaging.net.). disponible en: (30) National Comprehensive Cancer Network. NCCN Clinical Practice Guidelines in Oncology. Breast Cancer. NCCN V1; 2009.

11. TNM Classification of Malignant Tumours. 5th ed. (actualmente en vigor) United Estates of America: $1 . \mathrm{H}$ Sobin and Ch. Wittekind; 1997. Breast tumours; p.123-130.

12. Roca Maseda R, Perez Manga G. Valoración de la Incapacidad Laboral en Oncología. En: Instituto Nacional de Medicina y Seguridad del Trabajo. Guía de Valoración del Menoscabo Permanente, $2^{2}$ edición. Madrid:Longares I. y R. S.A.; 1999; Tomo III:274-275

13. Gerson R., Alban F., Villalobos A. and Serrano A. Recurrence and survival rates in early breast cancer cases with triple negative immunophenotype. Gaceta Médica de México 2008 144:1 (27-34)

14. Oncosur. Grupo de trabajo oncológico de centros hospitalarios del sur de Madrid. Guía clínica de diagnóstico y tratamiento del cáncer de mama. Madrid: YOU \& US, S.A.2009

15. Galván, A; Pons Y; Echevarría C ;Ibañez T. Patología postquirúrgica del cáncer de mama. Artículo especial. Rehabilitación (Madrid). 2008;Vol. 42(nº1)2008:27-33

16. Florez-García, MT; Valverde-Carrillo, MD. Eficacia del tratamiento conservador no farmacológico del linfedema postmastectomía. Rehabilitación (Madrid). 2007; Vol.4(nº3): 126-134.

17. Romero Culleres,G; Almendariz,A. Original. Linfedema después de cirugía por cáncer de mama. Estudio de incidencia y factores de riesgo en 113 pacioentes. Rehabilitación (Madrid). 2004;Vol.38(,nº 2):72-77

18. Ferlay J. Cancer Information Section, International Agency for Research on Cancer,,[sede web].Lyon, France: 2005 [actualizada noviembre 2008. Consultado 25/4/2010]. Disponible en; http://www-dep.iarc. fr/WHO_frame.htm.

19. Sociedad Española de Oncología [sede web]. Madrid. [acceso 20/4/2010/] Disponible en: wwwseom .org/ seommcms

||||||||||||||||||||||||||||||||||||||||||||||||||||||||||||||||||||||||||||||||||||||||||||||||||||||||||||||||||||||||||||||||||||||||||||||||||||||||||||||||||||||||||||||||||||||||||||||||||||||||||||||||||||||||||||||||| 